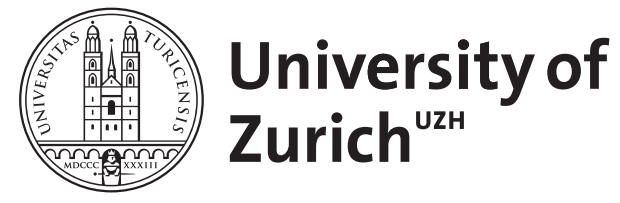
Archive

University of Zurich

University Library

Strickhofstrasse 39

CH-8057 Zurich

www.zora.uzh.ch

Year: 2017

\title{
The role of character traits in economic games
}

Ruch, Willibald ; Bruntsch, Richard ; Wagner, Lisa

DOI: https://doi.org/10.1016/j.paid.2016.12.007

Posted at the Zurich Open Repository and Archive, University of Zurich

ZORA URL: https://doi.org/10.5167/uzh-130329

Journal Article

Accepted Version

Originally published at:

Ruch, Willibald; Bruntsch, Richard; Wagner, Lisa (2017). The role of character traits in economic games. Personality and Individual Differences, 108:186-190.

DOI: https://doi.org/10.1016/j.paid.2016.12.007 
The role of character traits in economic games

Willibald Ruch, Richard Bruntsch, and Lisa Wagner

Department of Psychology, University of Zurich, Zurich, Switzerland,

Distance Learning University Switzerland, Brig, Switzerland.

Author Note

Willibald Ruch, Richard Bruntsch, and Lisa Wagner are at the Department of Psychology, University of Zurich, Switzerland, Binzmühlestrasse 14/7, 8050 Zurich, Switzerland, and at the Distance Learning University Switzerland, Brig, Switzerland.

Correspondence concerning this article should be addressed to: Willibald Ruch, Section on Personality and Assessment, Department of Psychology, University of Zurich, Binzmuehlestrasse 14 / Box 7, 8050 Zurich, Switzerland, E-mail:

w.ruch@psychologie.uzh.ch. Fax: +41 446357529. 


\begin{abstract}
Notwithstanding the fact that valuing personality descriptors from the realm of character were deliberately excluded from the Big Five model of personality, in the study of economic games (EGs; e.g., the prisoner's dilemma) the Big Five were used when predicting outcomes in the field of behaviors that are socially valued in terms of fairness and altruism (i.e., the moral domain). Eventually evaluative traits were introduced and found useful in the study of EGs, namely the HEXACO dimension honesty/humility $(\mathrm{H} / \mathrm{H})$. As an extensive list of evaluative traits, the Values In Action classification of character strengths may complement $\mathrm{H} / \mathrm{H}$ when assessing character and predicting individuals' decisions in EGs. For this study, $N$ $=155$ participants completed the Values in Action Inventory of Strengths, a measure of the HEXACO traits, and four different EGs that involved decisions relevant to fairness and altruism. Along with $\mathrm{H} / \mathrm{H}$, individuals' positions on a dimension abstracting heart-related vs. mind-related character strengths predicted outcomes in the EGs. These results support earlier findings that evaluative traits predict decisions relevant to fairness and altruism. Furthermore, character strengths can be seen as complementing $\mathrm{H} / \mathrm{H}$ when predicting behavior in the moral domain by character.
\end{abstract}

Keywords: Altruism, Character strengths, Economic games, Fairness, Honesty/humility, Moral domain, Personality 
The role of character traits in economic games

\section{Introduction}

Players tend to make fair and altruistic decisions in economic games like the prisoner's dilemma or the dictator game. This contradicts the notion of the homo oeconomicus, which posits that individuals' dominant interest is to rationally maximize their own economic benefit. It was demonstrated that deviations from strictly rational decisions are predicted by individuals' expressions of personality traits. Big Five personality traits such as neuroticism and foremost agreeableness were demonstrated to explain interindividual variance in economic game outcomes. While for example neuroticism is assumed to go along with a reduced tendency to take risks in the prisoner's dilemma (cf. Lönnqvist, Verkasalo, \& Walkowitz, 2011), Big Five agreeableness is assumed to be linked to decisions in economic games due to high scorers' higher (vs. low scorers' lower) motivation to promote positive interpersonal relationships (see Zhao \& Smillie, 2015, for an overview). While the role of the Big Five personality traits in economic games is well investigated, the introduction of honesty/humility $(\mathrm{H} / \mathrm{H})$ in the study of economic games was an important step toward predicting behavior in the moral domain by resorting to theoretically more relevant evaluative personality traits ${ }^{1}$. The term "moral domain" as used here is supposed to denote the field of behaviors that are socially esteemed in terms of virtue, goodness, and integrity, such as fairness and altruism. As a dimension of the HEXACO model of personality (cf. Ashton \& Lee, 2001) H/H can be seen as more closely and directly related to the concepts of fairness and altruism that play a role in individuals' decision in economic games (cf. Baumert, Schlösser, \& Schmitt, 2014). Across different economic games, H/H was found to be a predictor of players' decisions (e.g., Hilbig, Thielmann, Hepp, Klein, \& Zettler, 2015; Hilbig, Zettler, Leist, \& Heydasch, 2013). The inclusion of H/H in the HEXACO model of personality (Ashton \& Lee, 2001) was a great leap forward toward incorporating evaluative 
aspects in personality research. In the HEXACO model, five factors were found largely resembling the Big Five dimensions, while $\mathrm{H} / \mathrm{H}$ emerged as an additional dimension abstracting the covariance between evaluative descriptors with the facets sincerity, fairness, greed-avoidance, and modesty (cf. Ashton \& Lee, 2009). H/H can be seen as covering some of the very aspects of personality that were defined as character in terms of "personality evaluated" and distinguished from personality as temperament by early personality researchers (cf. Allport, 1921).

However, next to $\mathrm{H} / \mathrm{H}$ there is a comprehensive list of evaluative traits that can help to add to and complement $\mathrm{H} / \mathrm{H}$ when describing and classifying character, also in order to predict decisions in economic games and the moral domain in general. Peterson and Seligman (2004) proposed the VIA (Values in Action) classification of character strengths as positive traits that constitute the "good character". Character strengths are defined as ubiquitous, fulfilling, morally valued, trait-like, distinct and measurable individual differences (Peterson $\&$ Seligman, 2004). There are 24 character strengths that relate to six core virtues which are esteemed across cultures and historical epochs. The virtues and the related character strengths are: (1) wisdom and knowledge (includes the character strengths creativity, curiosity, openmindedness, love of learning, perspective), (2) courage (i.e., bravery, perseverance, honesty, zest), (3) humanity (i.e., capacity to love and be loved [short: love], kindness, social intelligence), (4) justice (i.e., teamwork, fairness, leadership), (5) temperance (i.e., forgiveness, modesty, prudence, self-regulation), and (6) transcendence (i.e., appreciation of beauty and excellence, gratitude, hope, humor, spirituality). A more detailed summary of the VIA classification is given by Ruch et al. (2010). Character strengths, as assessed with the established Values in Action Inventory of Strengths (VIA-IS; Peterson, Park, \& Seligman, 2005), were demonstrated to be stable across measurement points in terms of medium to high test-retest correlations in 3 to 9 months' intervals (e.g., Ruch et al., 2010). 
To attain an aggregated measure of two central aspects of the VIA-IS character strengths, an established can be used: Peterson (2006) suggested a two-dimensional factor space that consistently results from a principal component analysis of ipsatized scores of the character strengths. This approach classifies the entire character strengths on two axes: one bipolar dimension abstracts the covariance of "strengths of the mind (e.g., prudence, openmindedness) vs. strengths of the heart (e.g., zest, love)". The other bipolar dimension abstracts the covariance of character strengths relating to the self (e.g., creativity, love of learning) vs. strengths relating to others (e.g., teamwork, forgiveness). This ipsative approach was preferred over a normative one as in the economic games require decisions during which different strengths might be in conflict (e.g., kindness vs. open-mindedness). Hence, the decisions might be based on the relative rather than the absolute expression of the strengths.

The aim of the present paper is to test whether interindividual variance in economic game outcomes can be explained by individuals' expression of traits from the realm of evaluative personality description (i.e., $\mathrm{H} / \mathrm{H}$ and character strengths). Like in earlier studies on the role of $\mathrm{H} / \mathrm{H}$ in economic games (e.g., Hilbig et al., 2013, 2015), high expressions of $\mathrm{H} / \mathrm{H}$ are assumed to go along with fairer and more altruistic decisions than low expressions of $\mathrm{H} / \mathrm{H}$. Furthermore it is expected that individuals' location on two factorial dimensions of the VIA-IS predict decisions in economic games: as factor scores increase toward the "strengths of the heart" pole (e.g., zest, love) of the first dimension and to the "relating to others" pole (e.g., teamwork, forgiveness) of the second dimension, individuals are assumed to make fairer and more altruistic decisions. Accordingly, as a secondary objective of the present study, it was aimed at exploring whether character can be useful to gain a more detailed picture on who is inclined toward making fair and altruistic decisions (i.e., locating decision tendencies in economic games in the space spanned by two character trait dimensions representing the trade-offs between emotional vs. rational motives on the one hand and self- 
directed vs. other-directed motives on the other hand). To control for the effect of nonevaluative traits, all HEXACO dimensions other than $\mathrm{H} / \mathrm{H}$ will be included when predicting the decisions in the economic games from $\mathrm{H} / \mathrm{H}$ and the character strengths factors. As the VIA character strengths are reasoned to cover a broader spectrum of evaluative traits than $\mathrm{H} / \mathrm{H}$, they are expected to explain incremental variance in the economic game decisions beyond $\mathrm{H} / \mathrm{H}$.

\section{Method}

\subsection{Participants}

Participants were recruited via university mailing lists, psychology magazine websites, social platforms, and leaflets. In the advertisement, participants were offered a personal feedback on their individual expressions of character strengths and HEXACO personality traits. The sample included $N=155$ participants (33 males, age: $M=24.95$ years, $S D=7.61$ ). The majority of the participants were Swiss (75.5\%) or German (20.0\%). More than half $(55.5 \%)$ were currently students at a university or a university of applied sciences, $26.5 \%$ held a diploma allowing them to attend a university or a university of applied sciences, $14.2 \%$ completed a degree at a university or university of applied sciences, $1.9 \%$ completed vocational training, and $1.9 \%$ completed secondary education.

\subsection{Instruments}

The Values in Action Inventory of Strengths (VIA-IS; Peterson et al., 2005; German adaptation by Ruch et al., 2010) is a 240-item questionnaire for the assessment of the 24 character strengths (10 items per strengths) covered by the VIA classification (Peterson and Seligman, 2004). It uses a 5-point Likert-style scale ranging from 1 (“very much unlike me”) to 5 ("very much like me"). A sample item is "In a group, I try to make sure everyone feels 
included." (leadership). Several studies demonstrated the good psychometric properties of the German version of the VIA-IS (e.g., Proyer, Gander, Wellenzohn, \& Ruch, 2015). Internal consistencies in the present sample ranged from $\alpha=.70$ (teamwork) to .89 (creativity and spirituality) with a median of .76.

The HEXACO-60 (Ashton \& Lee, 2009) is a 60-item questionnaire for the assessment of the six dimensions of the HEXACO model of personality (10 items per dimension) employing a 5-point Likert-style scale ranging from 1 ("strongly disagree") to 5 ("strongly agree"). The six dimensions are: H/H (e.g., "I wouldn't use flattery to get a raise or promotion at work, even if I thought it would succeed.”), emotionality, extraversion, agreeableness, conscientiousness, and openness to experience. Internal consistencies in the present sample ranged from $\alpha=.69$ (agreeableness) to .83 (extraversion).

\subsubsection{Economic games}

On the basis of their payoff-rules, different economic games can be allocated loosely to one of two categories (cf. Zhao \& Smillie, 2015). Firstly, there are social dilemmas (characterized by a trade-off between more directly available attainment of outcomes relevant to the self-interest on the one hand and less securely available outcomes relevant to the collective interests on the other hand). Typically, individuals have the opportunity to choose either a "selfish" outcome that is a safe bet or a cooperative option that involves a leap of faith when taking a chance that the game partners might not simultaneously cooperate. That is, mutual cooperation leads to better overall outcomes while featuring a personal reward that is dependent from the other players' decisions (e.g., the prisoner's dilemma; cf. Dawes, 1980). The selfish outcome is typically designed as smaller but more warranted than the cooperative collective outcome that would result from all players expecting and performing mutual cooperation. As a second category, there are bargaining games, in which the players 
can allocate a share of a given amount of goods (e.g., money) to their game partners in a oneway allocation. Typically, in this category the payoff does not depend on the game partners' simultaneous contributions, although players may have the chance to refuse an offer in special variants (i.e., the ultimatum bargaining game) or may reciprocate or retaliate against previous offers when taking turns with a game partner in iterative rounds of games. Despite this distinction between two categories of economic games, a common denominator can be found by categorizing both types as involving conflicts between the interest of the self and concerns for others (so called mixed-motive situations, see Haesevoets, Folmer, \& Van Hiel, 2015). Accordingly, as a common feature, economic games involve decisions that are relevant to fairness and altruism (cf. Baumert et al., 2014; Zhao \& Smillie, 2015). In the economic games used in the present study, participants were instructed that they had been assigned to an anonymous game partner via a computer-assisted network that would be also keeping track of all game outcomes. If not otherwise specified, participants entered their offer by typing digits into a response field. To reduce the potential effect of different financial situations (i.e., income and savings), the games used play money (Talers), following the procedure suggested by Lönnqvist et al. (2011).

Prisoner's dilemma (adaptation by Lönnqvist et al., 2011). Participants` were to allocate zero up to ten Talers to their alleged game partner who would be asked to make the same decision as the participant. It was explained that participants' total outcome would depend both on their own decision and on their game partner's decisions. They were informed that the experimenter would double the allocated amount, so the best collective outcome would be if both allocated all their money. However, retaining one's own money and hoping for the game partner to allocate his or her money would have maximized the personal benefit. Participants entered their offer moving a slider bar with eleven steps (labeled “0” to “10”). 
Dictator Game (Kahneman, Knetsch, \& Thaler, 1986). Participants decided how many of a total of 100 Talers they wanted to allocate to their anonymous game partner. They were informed that they could keep as much as they wished.

Ultimatum Bargaining Game (Güth, Schmittberger, \& Schwarze, 1982). Participants decided how many of a total of 100 Talers they wanted to allocate to their anonymous game partner. They were instructed that the anonymous game partner would have the chance to refuse the share offer. Participants were informed that in this case they would lose their share.

Public Goods Game (cf. Kollock, 1998). Participants were instructed that they were playing with three game partners and they could anonymously contribute 0 up to 10 Talers to a shared account. The shared account would be doubled and equally distributed among the four game partners irrespective of their individual contribution. The best collective outcome would be attained if all four players allocated all their money. However, retaining one's own money and hoping for the game partners to allocate all their money would maximize the personal benefit when the money in the shared account is paid out.

\subsection{Procedure}

To avoid priming effects, the study was conducted in two sessions. In Session 1, participants answered the VIA-IS and the HEXACO-60 online. After one week they were invited to Session 2 via E-Mail and indicated their choices in the economic games online. The order of the economic games was randomized. As incentivized conditions were previously demonstrated to make associations between Big Five personality traits and outcomes of economic games become more evident (Lönnqvist et al., 2011), participants were given a real-world incentive to maximize the personal outcome. More specifically, participants were instructed that the more Talers they had at the end of the study the higher their chances would be to win one of ten online shopping vouchers amounting to 20 Swiss Franks in a prize draw. 
At the end of Session 2, participants were debriefed and informed that all participants had the same chances to win a prize.

\section{Data preparation}

The VIA-IS factor scores for the "strengths of the mind vs. strengths of the heart" and the "strengths relating to the self vs. strengths relating to others" dimension were derived by first ipsatizing the 24 scales (i.e., by subtracting the mean of all strengths from each single strength scale and dividing this difference by the individual standard deviation for each participant). Then, a principal component analysis was conducted on the intercorrelation of the ipsatized scales. Like in previous studies (e.g., Proyer et al., 2015; Ruch et al., 2010), two factors were extracted and obliquely rotated.

To obtain an aggregated measure of decision-making tendencies in economic games, in a first step the two game outcomes that ranged from 0 to 10 (Prisoner's Dilemma and Public Goods Game) were multiplied by 10 , so that the score can be interpreted as a percentage of the maximum amount. In a second step, an aggregated game score averaging the outcomes of the four games was generated. Higher (vs. lower) scores indicate decisions that are more in favor of the game partner or the common good. The four economic games as indicators for fair and altruistic tendencies yielded satisfactory convergence (mean $r=.29$; Cronbach's alpha $=.60)$ and the mean aggregated game score was $46.94(S D=17.74$, ranging from 0.00 to 87.50 ).

\section{Results}

The zero-order correlations between the aggregated game score and the other variables are given in Table 1. As Table 1 shows, the aggregated game score correlated with gender, $\mathrm{H} / \mathrm{H}$, the "strengths of the mind vs. heart" dimension of the VIA-IS. 
Insert Table 1 about here

To analyze if $\mathrm{H} / \mathrm{H}$ was a predictor of the economic game outcomes over and above the remaining five HEXACO traits (as a proxy for the Big Five) and to test whether the two dimensions of the VIA-IS had an incremental effect beyond all other predictors, a multiple regression analysis was conducted. Different predictors were entered in four consecutive steps in order to arrive at a hierarchical prediction of the economic game score as the criterion. That is, the respective predictor (or set of predictors) being looked at in one step of the analysis was added to the model on top of the predictors included in the previous step at any one time (naturally, except for Step 1). For example, the influences of $\mathrm{H} / \mathrm{H}$, the "Big Five" HEXACO traits, age, and gender were determined after the influences of the "Big Five" HEXACO traits, age, and gender were tested. Subsequently, the predictive value of the character strengths dimensions was jointly tested with all other predictors. This approach was preferred over the test of predictors in one single step in order to gain knowledge about the predictive power of the single (sets of) predictors in terms of an increment in the dependent variance explained by the model. The results are given in Table 2.

Insert Table 2 about here

As Table 2 shows, gender predicted the game score significantly in Step 1: women allocated more money to their game partners than men (overall model in Step 1: $F[3,152]=3.38, p=$ .037). In Step 2, the "Big Five" (i.e., the HEXACO dimension except for $\mathrm{H} / \mathrm{H}$ ) did not predict the game score significantly (overall model in Step 2: $F[7,147]=1.30, p=.256$ ). With $\mathrm{H} / \mathrm{H}$ entered in Step 3, $\mathrm{H} / \mathrm{H}$ emerged as a significant predictor and the overall model was significant $(F[8,146]=2.32, p=.023)$. In the final model $($ Step 4$), F(10,144)=2.54, p=$ .008 , the "strengths of the mind vs. strengths of the heart" dimension predicted the game 
score significantly beyond the influence of the HEXACO traits, of which again $\mathrm{H} / \mathrm{H}$ but also extraversion (with a negative weight) were significant predictors. The final model including all predictors explained 9\% (adjusted $R^{2}$ ) of the variance in the game score.

\section{Discussion}

As expected and line with previous findings, participants high in $\mathrm{H} / \mathrm{H}$ tended to allocate a greater amount of their game money to their game partners compared to participants low in $\mathrm{H} / \mathrm{H}$. The findings on extraversion have to be interpreted with strong reservation, as the influence of this variable is likely to be explained by a suppressor effect, as extraversion did not correlate with the game score on the bivariate level. Furthermore, as expected, participants scoring closer to the "heart" pole of the "strengths of the mind vs. strengths of the heart" dimension (as indicated by higher factor scores) allocated a greater amount of their game money to their game partners compared to participants scoring closer to the "mind" pole of this dimension (as indicated by lower factor scores). As indicated by a significant increment in the variance explained, character strengths can be seen as predicting the game score above and beyond the effect of $\mathrm{H} / \mathrm{H}$. This suggests that beyond sincerity, fairness, greed-avoidance, and modesty (i.e., the facets of $\mathrm{H} / \mathrm{H}$ ) the VIA classification can help to describe, classify, and assess character in a more exhaustive fashion.

Contrary to expectations, the dimension "strengths relating to the self vs. strengths relating to others" did not explain variance in the game outcomes significantly. It seems that the "mind vs. heart" dimension abstracted all the covariance among the character strengths that is relevant to decisions in economic games. Hence, it can be suggested that rather than driven by a trade-off between the focus on the self and the focus on others, fair and altruistic acts can be explained by decisions that are "rational vs. affectionate" with "heart-spirited" individuals allocating more money to their game partners than "mind-driven" individuals in 
the economic games. Hence, as we also aimed at defining the usefulness of character in the moral domain by developing a more detailed picture on who is inclined toward making fair and altruistic decisions, we may suggest that economic games — and maybe also moral decisions in general - are a matter of compassionate emotional involvement rather than merely a mixed-motive situation characterized by a conflict of the interest of the self vs. concerns for others (cf. Haesevoets et al., 2015).

The results suggest that as a comprehensive collection of evaluative traits the VIA classification of character strengths is worth considering when explaining variance in players' decisions in economic games operationalizing fairness and altruism — along with (and maybe even beyond) a second established conceptualization of character, i.e., HEXACO H/H. While $\mathrm{H} / \mathrm{H}$ was developed using a lexical approach, the VIA classification was deducted from various sources dealing with virtue and the "good character" (such as boy scout statutes or philosophers' writings) and hence almost naturally covers a broader spectrum of evaluative traits.

Considering earlier findings on the role of the Big Five in economic games, it is may seem surprising that the "Big Five" traits as entailed in the HEXACO (i.e., the "EXACO") did not have any predictive value for the game score, apart from the effect of extraversion (which may be based on a suppressor effect). However, this result is consistent with previous findings indicating no significant correlations between the "EXACO" and the allocations in a one-shot dictator game whereas some of the traditional Big Five measures were correlated significantly (Hilbig et al., 2015). There are at least two possible explanations for this. Firstly, the "EXACO" dimensions do not exactly replicate the traditional Big Five. Because of extracting an additional factor, in the HEXACO model the aspects relevant for decisions in economic games may be moved off from the five factors corresponding to the Big Five and 
attached to $\mathrm{H} / \mathrm{H}$. Secondly, the role of the "EXACO" traits in economic games may be underestimated when looking only at one-shot paradigms rather than iterative games. For example, agreeableness as entailed in the HEXACO model may come into play only after an individual did not encounter reciprocity in previous rounds with highly agreeable individuals tending to indulge being exploited. In support of this assumption, previous findings indicate that agreeableness (as entailed in the HEXACO model) is linked to not retaliating in the ultimatum bargaining game in terms of a "reactive" cooperation (Hilbig et al., 2013).

The present study may be limited by the relatively small sample, which included more female than male participants. Even though we statistically controlled for the effects of gender, it would be desirable for future studies to include a gender-balanced sample that also allows for a more systematic investigation of gender differences. Another limitation of the present study might be that, similar to previous studies (e.g., Hilbig et al., 2015), a relatively short (60-item) measure of the HEXACO traits was used. Most likely, the full version captures more aspects of the facets relevant for predicting behavior in economic games than the short version. Hence, $\mathrm{H} / \mathrm{H}$ might explain even more variance in economic games than the present findings indicate. In other words, the incremental variance explained by the VIA-IS might be somewhat overestimated. However, if the given assumption holds true, it is also possible that the joint predictive power of all evaluative traits measured (i.e., $\mathrm{H} / \mathrm{H}$ and character strengths) is underestimated in this study.

\subsection{Conclusions}

The present findings indicate that evaluative traits (i.e., $\mathrm{H} / \mathrm{H}$ and character strengths) as aspects of character are useful when predicting decisions in the moral domain. The VIA classification complements personality description in the evaluative spectrum and-in the 
light of the present finding — can be seen as adding to the $\mathrm{H} / \mathrm{H}$ dimension when accounting for behavior that is socially esteemed in terms of virtue, goodness, and integrity.

\section{Acknowledgements:}

This research did not receive any specific grant from funding agencies in the public, commercial, or not-for-profit sectors. The authors would like to thank Isabelle Keller and Melanie Simic for their help during data collection. 


\section{References}

Allport, G. W. (1921). Personality and character. Psychological Bulletin, 18, 441-445. doi:10.1037/h0066265.

Almagor, M., Tellegen, A., \& Waller, N. G. (1995). The Big Seven model: A cross-cultural replication and further exploration of the basic dimensions of natural language trait descriptors. Journal of Personality and Social Psychology, 69, 300-307. doi:10.1037/0022-3514.69.2.300

Ashton, M. C., \& Lee, K. (2001). A theoretical basis for the major dimensions of personality. European Journal of Personality, 15, 327-353. doi:10.1002/per.417

Ashton, M. C., \& Lee, K. (2009). The HEXACO-60: A short measure of the major dimensions of personality. Journal of Personality Assessment, 91, 340-345. doi:10.1080/00223890902935878

Baumert, A., Schlösser, T., \& Schmitt, M. (2014). Economic games: A performance-based assessment of fairness and altruism. European Journal of Psychological Assessment, 30, 178-192. doi:10.1027/1015-5759/a000183

Dawes, R. M. (1980). Social dilemmas. Annual Review of Psychology, 31, 169-193. doi:10.1146/annurev.ps.31.020180.001125

De Raad, B., \& Barelds, D. P. H. (2008). A new taxonomy of Dutch personality traits based on a comprehensive and unrestricted list of descriptors. Journal of Personality and Social Psychology, 94, 347-364. doi:10.1037/0022-3514.94.2.347

Güth, W., Schmittberger, R., \& Schwarze, B. (1982). An experimental analysis of ultimatum bargaining. Journal of Economic Behavior \& Organization, 3, 367-388. doi:10.1016/0167-2681(82)90011-7. 
Haesevoets, T., Folmer, C. R., \& Van Hiel, A. (2015). Cooperation in mixed-motive games: The role of individual differences in selfish and social orientation. European Journal of Personality, 29, 445-458. doi:10.1002/per.1992

Hilbig, B. E., Zettler, I., Leist, F., \& Heydasch, T. (2013). It takes two: Honesty-Humility and Agreeableness differentially predict active versus reactive cooperation. Personality and Individual Differences, 54, 598-603. doi:10.1016/j.paid.2012.11.008

Hilbig, B. E., Thielmann, I., Hepp, J., Klein, S. A., \& Zettler, I. (2015). From personality to altruistic behavior (and back): Evidence from a double-blind dictator game. Journal of Research in Personality, 55, 46-50. doi:83010.1016/j.jrp.2014.12.004

Kahneman, D., Knetsch, J. L., \& Thaler, R. H. (1986). Fairness and the assumptions of economics. The Journal of Business, 59, 285-300.

Kollock, P. (1998). Social dilemmas: the anatomy of cooperation. Annual Review of Sociology, 24, 183-214.

Lönnqvist, J. E., Verkasalo, M., \& Walkowitz, G. (2011). It pays to pay-Big Five personality influences on co-operative behaviour in an incentivized and hypothetical prisoner's dilemma game. Personality and Individual Differences, 50, 300-304. doi:10.1016/j.paid.2010.10.009

Peterson, C., \& Seligman, M. E. P. (2004). Character strengths and virtues: A handbook and classification. Washington, DC: American Psychological Association.

Peterson, C., Park, N., \& Seligman, M. E. P. (2005). Assessment of character strengths. In G. P. Koocher, J. C. Norcross, \& S. S. Hill III (Eds.), Psychologists' desk reference (2nd ed., pp. 93-98). New York: Oxford University Press. 
Peterson, C. (2006). A primer in positive psychology. New York: Oxford University Press.

Proyer, R. T., Gander, F., Wellenzohn, S., \& Ruch, W. (2015). Strengths-based positive psychology interventions: A randomized placebo-controlled online trial on long-term effects for a signature strengths- vs. a lesser strengths-intervention. Frontiers in Psychology, 6. 456. doi:10.3389/fpsyg.2015.00456

Ruch, W., Proyer, R. T., Harzer, C., Park, N., Peterson, C., \& Seligman, M. E. P. (2010). Values in Action Inventory of Strengths (VIA-IS): Adaptation and validation of the German version and the development of a peer-rating form. Journal of Individual Differences, 31, 138-149. doi:10.1027/1614-0001/a000022

Zhao, K., \& Smillie, L. D. (2015). The role of interpersonal traits in social decision making: Exploring sources of behavioral heterogeneity in economic games. Personality and Social Psychology Review, 19, 277-302. doi:10.1177/1088868314553709 


\section{Footnotes}

1 Evaluative trait descriptors were deliberately excluded from the item pools analyzed in the course of the development of the Big Five model (cf. Almagor, Tellegen, \& Waller, 1995). Studies using more inclusive and lexically more representative item samples support the notion that, beyond the Big Five, additional dimensions exist that abstract socially valued aspects of personality in terms of personality evaluated according to prevailing standards of conduct (cf. Allport, 1921; i.e., Ashton \& Lee, 2001: H/H; Almagor et al., 1995: positive valence and negative valence; De Raad \& Barelds, 2008: virtue, competence, and hedonism). 
Table 1

Correlations Between the Economic Game Score, Age, Gender, HEXACO Traits, and Character Strengths Factors

\begin{tabular}{|c|c|c|c|c|c|c|c|c|c|c|}
\hline & $(2)$ & (3) & (4) & $(5)$ & (6) & (7) & (8) & (9) & (10) & (11) \\
\hline (1) Economic Game Score & .01 & $.21 *$ & $.25^{*}$ & .00 & -.01 & .05 & -.06 & -.03 & $.17^{*}$ & .11 \\
\hline (2) Age & & -.02 & $.20^{*}$ & -.06 & .03 & .12 & .00 & .15 & -.07 & .01 \\
\hline (3) Gender & & & $.22 *$ & $.27^{*}$ & $.17^{*}$ & .06 & .09 & -.08 & $.27^{*}$ & $.29 *$ \\
\hline (4) Honesty-Humility & & & & -.04 & $.27 *$ & $.34 *$ & .11 & -.05 & .13 & $.28^{*}$ \\
\hline (5) Emotionality & & & & & $-.23 *$ & -.08 & .14 & $-.22 *$ & -.05 & $.51^{*}$ \\
\hline (6) Extraversion & & & & & & .13 & -.05 & .02 & $.66^{*}$ & -.05 \\
\hline (7) Agreeableness & & & & & & & .01 & -.01 & .01 & $.36^{*}$ \\
\hline (8) Conscientiousness & & & & & & & & -.10 & $-.26^{*}$ & .04 \\
\hline (9) Openness & & & & & & & & & -.04 & $-.43 *$ \\
\hline (10) Strengths of the mind vs. heart & & & & & & & & & & .06 \\
\hline
\end{tabular}

Note. $N=155$. Gender: $0=$ male, $1=$ female.

$* p<.05$ (two-tailed). 
Table 2

Hierarchical Regression Analysis Predicting the Total Score Across Four Economic Games

\begin{tabular}{|c|c|c|c|c|}
\hline & $B$ & $S E B$ & $\beta$ & $\Delta R^{2}$ \\
\hline Step 1: Demographic variables & & & & $.04 *$ \\
\hline Age & 0.03 & 0.19 & .01 & \\
\hline Gender $(0=$ male, $1=$ female $)$ & 8.91 & 3.43 & $.21^{*}$ & \\
\hline Step 2: HEXACO except for Honesty/Humility & & & & .02 \\
\hline Age & 0.02 & 0.19 & .01 & \\
\hline Gender $(0=$ male, $1=$ female $)$ & 10.39 & 3.72 & $.24 *$ & \\
\hline Emotionality & -2.34 & 2.83 & -.07 & \\
\hline Extraversion & -2.08 & 2.42 & -.07 & \\
\hline Agreeableness & 1.51 & 2.93 & .04 & \\
\hline Conscientiousness & -2.75 & 2.85 & -.08 & \\
\hline Openness & -1.06 & 2.46 & -.04 & \\
\hline Step 3: HEXACO Honesty/Humility & & & & $.05^{*}$ \\
\hline Age & -0.09 & 0.19 & -.04 & \\
\hline Gender $(0=$ male, $1=$ female $)$ & 8.53 & 3.67 & $.20 *$ & \\
\hline Emotionality & -2.07 & 2.76 & -.07 & \\
\hline Extraversion & -3.56 & 2.41 & -.13 & \\
\hline Agreeableness & -1.14 & 2.99 & -.03 & \\
\hline Conscientiousness & -3.64 & 2.80 & -.10 & \\
\hline Openness & -0.55 & 2.40 & -.02 & \\
\hline Honesty/Humility & 7.50 & 2.50 & $.27^{*}$ & \\
\hline Step 4: Character strengths dimensions & & & & $.04 *$ \\
\hline Age & -0.04 & 0.19 & -.02 & \\
\hline Gender $(0=$ male, $1=$ female $)$ & 6.47 & 3.75 & .15 & \\
\hline
\end{tabular}




\begin{tabular}{llll}
\hline Emotionality & -2.70 & 3.19 & -.09 \\
Extraversion & -8.78 & 3.17 & $-.31^{*}$ \\
Agreeableness & -0.57 & 3.28 & -.02 \\
Conscientiousness & -1.11 & 2.95 & -.03 \\
Openness & -0.19 & 2.63 & -.01 \\
Honesty/Humility & 7.65 & 2.53 & $.27^{*}$ \\
Strengths of the mind $(-)$ vs. heart $(+)$ & 5.04 & 2.01 & $.28^{*}$ \\
Strengths relating to the self $(-)$ vs. others $(+)$ & 0.00 & 2.09 & .00
\end{tabular}

Note. $N=155$. Total $R^{2}=.15$. Total adjusted $R^{2}=.09$. Method: enter.

$* p<.05$ (two-tailed). 